\title{
Multiple pulmonary emboli as a result of renal cell carcinoma: A case report
}

\author{
BING LI, HONG ZENG, MEI DING, PING YANG and YUQUAN HE \\ Department of Cardiology, China-Japan Union Hospital of Jilin University, Changchun, Jilin 130033, P.R. China
}

Received February 19, 2015; Accepted March 10, 2016

DOI: $10.3892 / \mathrm{ol} .2016 .5398$

\begin{abstract}
Pulmonary embolism is the most prevalent and potentially fatal complication of deep vein thrombosis. Renal cell carcinoma (RCC) is occasionally associated with pulmonary embolism, occurring as a result of secondary hypercoagulable states or cancer-associated emboli. The current study describes the case of a 43-year-old male that experienced 'squeezing' pain in the chest, considered to mimic acute coronary syndrome. Following a number of diagnostic examinations, the patient was diagnosed with pulmonary embolism, which led to the detection of RCC. The patient did not experience any symptoms until the diagnosis of pulmonary embolism, therefore the asymptomatic tumor was considered as one of the possible causes. Abdominal ultrasonography examination is highly recommended for screening in all patients, particularly those that exhibit uncharacteristic symptoms, to improve the detection and diagnosis of RCC and associated pulmonary emboli.
\end{abstract}

\section{Introduction}

Chest pain is a common clinical symptom resulting from various conditions, including trauma, acute coronary syndrome, malignant tumors and aortic dissection (1-4). Therefore, misdiagnosis may easily occur and a pulmonary embolism developing as a result of an asymptomatic malignant tumor may go undetected. The current study describes the case of a 43-year-old male, who was referred to the Department of Cardiology, China-Japan Union Hospital of Jilin University (Changchun, China) presenting with sudden 'squeezing' pain in the chest and dyspnea. The symptoms were suspected to be associated with a possible pulmonary embolism. The patient was later diagnosed with multiple pulmonary emboli occurring as a result of renal cell carcinoma (RCC).

Correspondence to: Dr Ping Yang or Dr Yuquan He, Department of Cardiology, China-Japan Union Hospital of Jilin University, 126 Xiantai Street, Changchun, Jilin 130033, P.R. China

E-mail: pyang@jlu.edu.cn

E-mail: hyq2@sina.com

Key words: pulmonary embolism, renal cell carcinoma, asymptomatic tumor
Almost 58,000 new cases of RCC were expected in the USA in 2009, and the morbidity rate is increasing each year. As the increase in the number of diagnoses is not solely due to early detection, the mortality rate of RCC is high and exhibits no decreasing trend. Currently, RCC is the 7th most common cancer in men and the 8th most common cancer in women in the USA (5). The chest pain due to pulmonary emboli may be an initial symptom of RCC.

\section{Case report}

In December 2012, a 43-year-old male presented to the Department of Cardiology, China-Japan Union Hospital of Jilin University, complaining of sudden 'squeezing' pain in the chest and dyspnea. The medical history of the patient was unremarkable with no history of trauma, no family history of renal tumors and no symptoms of hematuria or abdominal pain. The patient had a smoking history of 25 years with an average of 140 cigarettes per week. Physical examination reported a blood pressure reading of 100/80 $\mathrm{mmHg}$ (nomal range, $139-90 / 99-60 \mathrm{mmHg}$ ) and a heart rate of 88 beats/min (normal range, 60-100 beats/min). An electrocardiogram (ECG) identified oblique type ST segment elevation in leads $\mathrm{V} 1-\mathrm{V} 3$. Troponin was elevated to $0.85 \mathrm{ng} / \mathrm{ml}$ (normal range, 0-0.04 ng/ml), and other myocardial biomarker levels were normal.

The initial suspected diagnosis was acute coronary syndrome, primarily due to the characteristic changes observed in the ECG and the markedly elevated myocardial biomarker level. Emergency coronary angiography showed normal coronary arteries. The possibility of pulmonary embolism or aortic dissection was not excluded; however, the patient had no history of hypertension and the current blood pressure was within normal limits, thereby reducing the likelihood of aortic dissection.

Pulmonary embolism-associated laboratory and auxiliary examinations were subsequently performed and the results were as follows: D-dimer, 4,162 ng/ml (normal range, 0-400 ng/ml); blood gas analysis: $\mathrm{pH}, 7.42$ (normal range, 7.35-7.45); $\mathrm{pCO}_{2}, 35 \mathrm{mmHg}$ (normal range, 35-45 mmHg); $\mathrm{pO}_{2}, 72 \mathrm{mmHg}$ (normal range, $80-100 \mathrm{mmHg}$ ); and base excess, $2.5 \mathrm{mmol} / \mathrm{l}$ (-3.0 to $3.0 \mathrm{mmol} / \mathrm{l})$. Pulmonary ventilation/perfusion scintigraphy identified multiple pulmonary emboli in each lung (Fig. 1). Furthermore, computed tomography angiography (CTA) revealed pulmonary emboli 

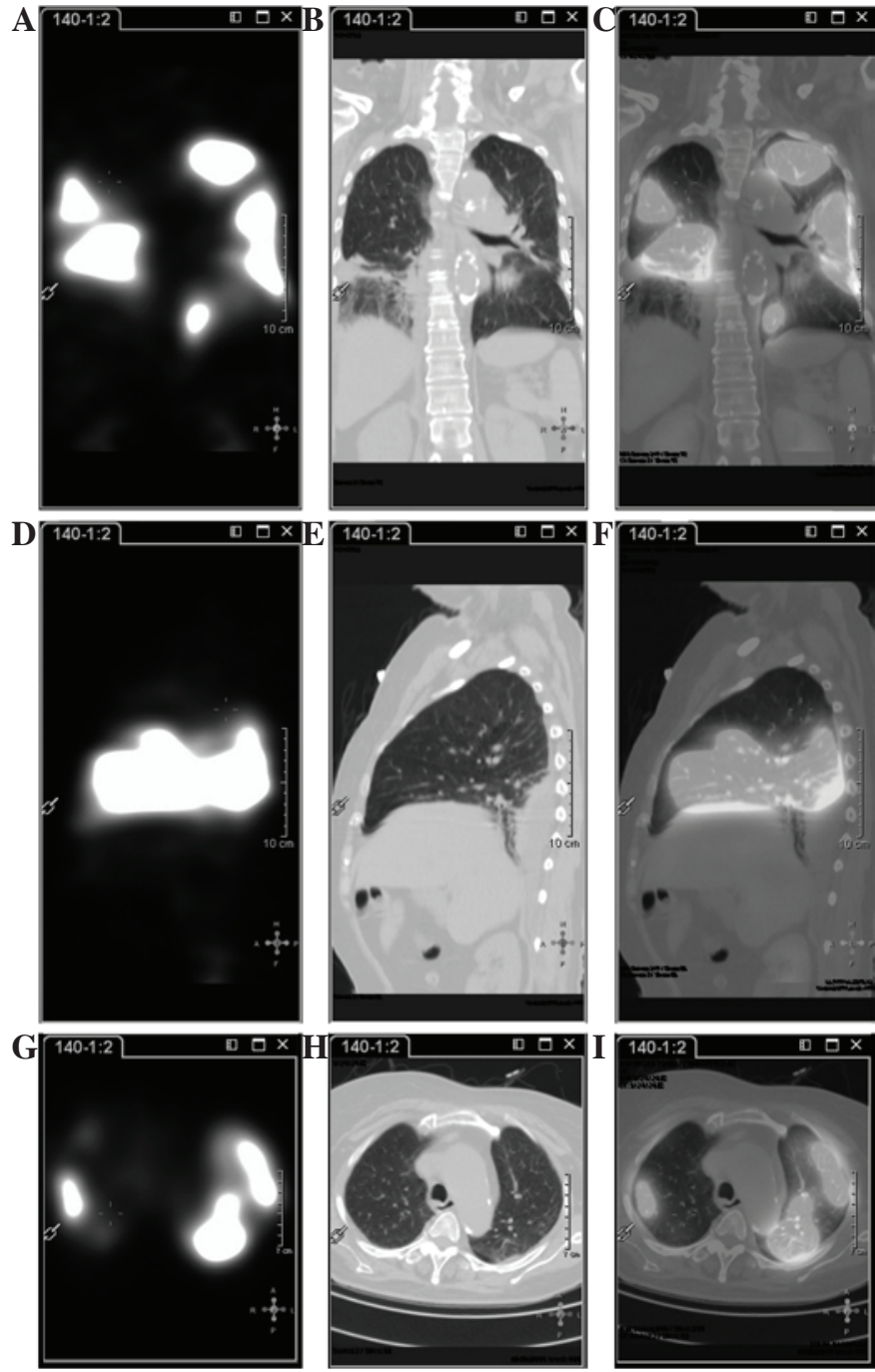

Figure 1. Pulmonary ventilation/perfusion scintigraphy images (A-C) Coronal, (D-F) sagittal and (G-I) axial images showing multiple irregular distribution of radioactive reduced anomalies in each lobe of each lung, particularly the superior lobes.

over each lung field and pulmonary emphysema of the left lung (Fig. 2), prompting a diagnosis of pulmonary embolism.

Generally, $70 \%$ of pulmonary emboli occur as a result of lower limb deep vein thrombosis $(6,7)$. However, the patient in the present case had normal lower limb ultrasonography results. Routine abdominal ultrasonography examination identified a substantial, heterogeneous, hypoechoic lesion, and subsequent enhanced magnetic resonance imaging (MRI) of the kidney revealed a massive shadow $(3.9 \times 5.1 \times 4.2 \mathrm{~cm})$ in the upper pole of the left kidney (Fig. 3) with a lower density, patchy, necrotic area in the center. In addition, MRI identified that the inferior vena cava and proximal region of the left kidney vein were significantly dilated with multiple filling defects within the cavities. The renopuncture tissues were used for SP staining and immunohistochemical analysis with monoclonal mouse anti-human Ki-67 antigen (clone MIB-1; Agilent Technologies Co. Ltd., Beijing, China). The result showed that the pathological change was consistent with RCC, cytonecrosis and worse degeneration. There was a Ki-67 index of $20 \%$. When combined with the other test results, a diagnosis of stage III (T3bNOM0) RCC (8) was formed. The

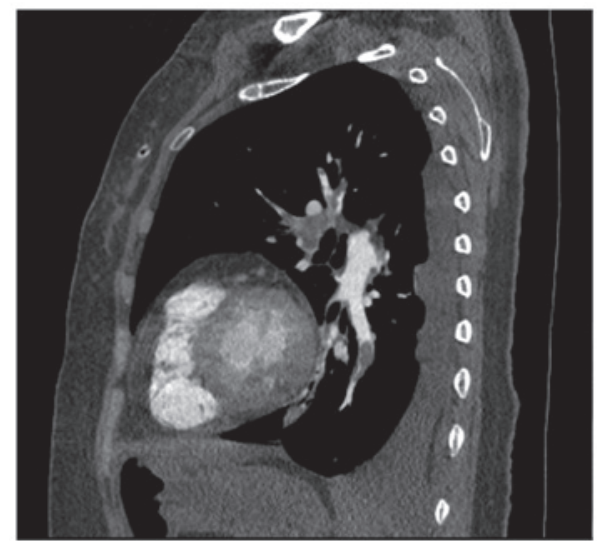

Figure 2. Pulmonary computed tomography angiography image showing an intraluminal bar filling defect in the pulmonary artery of the lower lobe of the left lung.

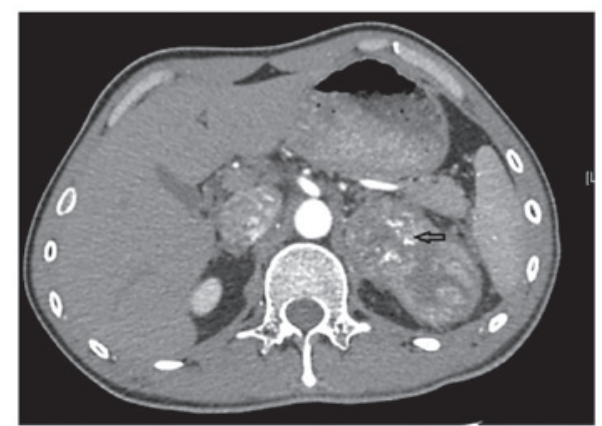

Figure 3. Magnetic resonance imaging of the kidney showing a 3.9x $5.1 \times 4.2-\mathrm{cm}$ mass with heterogeneous enhancement, an unclear boundary and low-density necrosis in the left kidney on enhanced arterial phase. The arrow indicates the tumor.

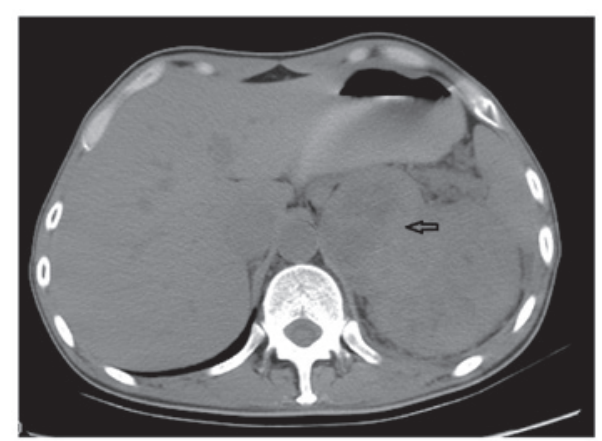

Figure 4. Renal computed tomography at the 1 year follow-up showing a mass with heterogeneous density, an unclear boundary and low-density necrosis in the left kidney, which is $12.1 \mathrm{~cm}$ in diameter. The arrow indicates the tumor.

patient did not undergo surgery due to the risk of bleeding from large vessels present near the tumor, and was instead treated with oral warfarin $(1.25 \mathrm{mg}$; once a day for $1 \mathrm{month})$ and subcutaneous injections of low molecular weight heparin ( $40 \mathrm{mg}$; twice daily for 7 days), which significantly alleviated symptoms in comparison with pretreatment.

In addition, sunitinib targeted therapy was initiated for 1 month, and regular chemotherapy with interleukin-2 was administered, consisting of 600,000 intravenous units per 
day for 10 days and 2 million units via aerosol inhalation per day for 10 days every 3 months. At 12 months after the initial treatment, the patient began to experience left abdominal pain and the maximal diameter of the left renal mass had increased by $7 \mathrm{~cm}$ (12.1 cm in diameter) on renal CT images. Furthermore, multiple small lymph nodes were identified in the abdomen (Fig. 4). At this time the patient had poor general health, and exhibited shortness of breath and chest congestion during limited physical activity that required frequent oxygen inhalation. Since surgery could not be performed and targeted therapy using sunitinib was not desired, the prognosis of the patient is poor. A follow-up was planned every 6 months.

Written informed consent was obtained from the patient for the publication of the present study.

\section{Discussion}

In the present case, a 43-year-old male presented with severe chest pain, and a diagnosis of acute coronary syndrome was initially suspected due to the excruciating chest pain, ischemic ECG changes and an elevated myocardial biomarker. However, negative coronary angiogram results excluded coronary artery abnormalities. With regards to early clinical manifestation and auxiliary examination results, one-third of patients with an acute pulmonary embolism demonstrate uncharacteristic features that may mimic acute coronary syndrome, resulting in a false diagnosis (6). It is widely known that up to $70 \%$ of pulmonary emboli occur as a complication of lower limb deep vein thrombosis, and ultrasonography examination is the first choice modality for screening venous thrombi. However, abdominal ultrasonography can also be useful for screening venous thrombi $(9,10)$. In the present case, the asymptomatic, large, renal tumor was clearly identified by abdominal ultrasonography examination as a space-occupying phenomenon in the left renal area.

The early symptoms of RCC are uncharacteristic and vary among individuals, which often leads to false and/or misdiagnosis (10). Only $10 \%$ of patients with RCC present with the three characteristic clinical symptoms observed during early stages, including hematuria, abdominal pain and an abdominal mass, whereas $>40 \%$ of patients do not present with these symptoms (10). In the current case, the patient lacked the typical symptoms of RCC and primarily presented with symptoms of pulmonary embolism.

Cancer is one of the leading causes of pulmonary embolism, however, asymptomatic malignant tumors are not always found in the clinic (11). Previous studies have demonstrated that $10 \%$ of patients with idiopathic or unexplained pulmonary emboli were subsequently diagnosed with malignant tumors at 5-10 year follow-ups, indicating the requirement for greater attention to secondary pulmonary emboli caused by asymptomatic tumors (11-13).

Thromboembolism may occur in patients with cancer as a result of hypercoagulable states and is closely associated with degree of malignancy, tumor location, pathological type and tumor staging (14). In the present case, the renal tumor was solid, highly malignant and may have metastasized to distant organs through the blood stream, which is an important risk factor for the occurrence of pulmonary embolism from thrombosis. In addition, the RCC invaded the inferior vena cava and the proximal region of the left kidney vein, resulting in vascular injuries, inflammatory response and vascular stenosis, thereby promoting thrombogenesis.

The pulmonary ventilation/perfusion scintigraphy and pulmonary CTA showed highly consistent results when compared with the elevated D-dimer results, but the pulmonary ventilation/perfusion scintigraphy was more meaningful for the diagnosis of pulmonary embolism below subsegmental levels. In comparison with ventilation/perfusion scintigraphy, pulmonary CTA has a higher diagnostic sensitivity and specificity, and a higher accuracy in identifying the attached embolismic sites (3). A total of $92-96 \%$ of positive diagnostic results have been diagnosed following CTA in patients with an intermediate or highly suspected pulmonary embolism (3).

In the current case, dyspnea and markedly restricted physical activities reoccurred at the 1 year follow-up, indicating recurrence of the pulmonary embolism, which most likely resulted from insufficient anticoagulation intensity and duration. However, the possibility of cancer-associated emboli could not be excluded. Progressive targeted therapies for the treatment of RCC and other comprehensive supporting strategies should be administered in combination with long-term anticoagulants for concurrent pulmonary embolism. Such therapy aims to improve the patient prognosis and enhance the quality of life.

In conclusion, $\mathrm{RCC}$ is a common malignant tumor that involves the urinary system and is associated with a high mortality rate. Given that only $10 \%$ of patients present with the three characteristic clinical symptoms, it is difficult to accurately diagnose the disease in the early stages. Therefore, clinicians should pay close attention to patients presenting with uncharacteristic symptoms and strengthen the early screening process for RCC based on ultrasonography or CT examinations.

\section{Acknowledgements}

This study was supported by a grant from the National Science Foundation entitled 'function and mechanism of the activin A/FS system imbalance in the myocardial cell apoptosis of heart failure' (grant no. 81270315).

\section{References}

1. Hamm CW, Bassand JP, Agewall S, Bax J, Boersma E, Bueno H, Caso P, Dudek D, Gielen S, Huber K, et al; ESC Committee for Practice Guidelines: ESC Guidelines for the management of acute coronary syndromes in patients presenting without persistent ST-segment elevation: The Task Force for the management of acute coronary syndromes (ACS) in patients presenting without persistent ST-segment elevation of the European Society of Cardiology (ESC). Eur Heart J 32: 2999-3054, 2011.

2. Task Force on the management of ST-segment elevation acute myocardial infarction of the European Society of Cardiology (ESC); Steg PG, James SK, Atar D, Badano LP, Blömstrom-Lundqvist C, Borger MA, Di Mario C, Dickstein K, Ducrocq G, Fernandez-Aviles F, et al: ESC Guidelines for the management of acute myocardial infarction in patients presenting with ST-segment elevation. Eur Heart J 33: 2569 2619, 2012.

3. Torbicki A, Perrier A, Konstantinides S, Agnelli G, Galiè N, Pruszczyk P, Bengel F, Brady AJ, Ferreira D, Janssens U, et al; ESC Committee for Practice Guidelines (CPG): Guidelines on the diagnosis and management of acute pulmonary embolism: The task force for the diagnosis and management of acute pulmonary embolism of the European society of cardiology (ESC). Eur Heart J 29: 2276-2315, 2008. 
4. Nienaber CA and Powell JT: Management of acute aortic syndromes. Eur Heart J 33: 26-35b, 2012.

5. Amerian Cancer Society: Cancer Facts and Figures 2009. American Cancer Society, Atlanta, USA, 2009.

6. Kukla P, Długopolski R, Krupa E, Furtak R, Mirek-Bryniarska E, Szełemej R, Jastrzębski M, Nowak J, Kulak L, Hybel J, et al: How often pulmonary embolism mimics acute coronary syndrome? Kardiol Pol 69: 235-240, 2011.

7. Dalen JE: Pulmonary embolism: What have we learned since Virchow? Natural history, pathophysiology, and diagnosis. Chest 122: 1440-1456, 2002.

8. Decastro GJ and McKiernan JM: Epidemiology, clinical staging, and presentation of renal cell carcinoma. Urol Clin North Am 35: 581-592, 2008

9. Kearon C: Natural history of venous thromboembolism. Circulation 107 (23 Suppl 1): I22-I30, 2003.
10. Gibbons RP, Monte JE, Correa RJ Jr and Mason JT: Manifestations of renal cell carcinoma. Urology 8: 201-206, 1976.

11. Monreal M, Fernandez-Llamazares J, Perandreu J, Urrutia A Sahuquillo JC and Contel E: Occult cancer in patients with venous thromboembolism: Which patients, which cancers. Thromb Haemost 78: 1316-1318, 1997.

12. Hettiarachchi RJ, Lok J, Prins MH, Büller HR and Prandoni P: Undiagnosed malignancy in patients with deep vein thrombosis: Incidence, risk indicators, and diagnosis. Cancer 83: 180-185, 1998.

13. Schulman S and Lindmarker P: Incidence of cancer after prophylaxis with warfarin against recurrent venous thromboembolism. Duration of Anticoagulation Trial. N Engl J Med 342: 1953-1958, 2000

14. Connolly GC and Francis CW: Cancer-associated thrombosis. Hematology Am Soc Hematol Educ Program 2013: 684-691, 2013. 